\title{
Gigantomastia During Pregnancy Due to Burkitt Lymphoma
}

\author{
(D) Virginia Foreste ${ }^{1}$, (D) Luigi Della Corte ${ }^{1}$, (D) Cristina Stradella ${ }^{1}$, (D) Bianca Cusati ${ }^{2}$, (D) Guido Coco $^{3}$, (D) Luigi Stradella ${ }^{4}$ \\ ${ }^{1}$ Department of Neuroscience, University of Naples Federico II, Naples, Italy, Reproductive Sciences and Dentistry, School of Medicine, Napoli, Italy \\ ${ }^{2}$ Department of Department of Diagnostic Imaging, P.O. Santa Maria delle Grazie Asl Napoli 2 Nord., Napoli, Italy \\ ${ }^{3}$ Department of Oncology and Breast Surgery, P.O. Santa Maria delle Grazie Asl Napoli 2 Nord, Napoli, Italy \\ ${ }^{4}$ Department of Obstetrics and Gynecology, P.O. Santa Maria delle Grazie Asl Napoli 2 Nord, Napoli, Italy
}

\section{ABSTRACT}

Gigantomastia is a rare complication of pregnancy usually associated with benign conditions and rarely with malignancies. This paper reports a non-Hodgkin lymphoma case associated with gigantomastia during pregnancy. The patient was a 30-year-old gravida one woman, with a history of rapidly enlarging right breast at 2 weeks prior to presentation. After the first diagnosis of benign gigantomastia, the continuous growth of the breast, despite the delivery and bromocriptine therapy, required further investigation of the case. The histological analysis revealed the presence of Burkitt lymphoma. Malignant causes of unilateral gigantomastia in pregnancy should be considered in the differential diagnosis of this condition.

Keywords: Breast tumor, Burkitt lymphoma, gigantomastia

Cite this article as: Foreste V, Della Corte L, Stradella C, Cusati B, Coco G, Stradella L. Gigantomastia During Pregnancy Due to Burkitt Lymphoma. Eur J Breast Health 2021; 17(1): 76-79.

\section{Introduction}

Gigantomastia is a rare complication of pregnancy usually associated with benign conditions, with an estimated incidence of 1 per $28,000-$ 100,000 pregnancies. It is defined as a diffuse increase of the breast size often leading to pitting edema, necrosis, hemorrhage, and ulcerations. Although the etiology is often undetermined, end-organ hypersensitivity to normal hormone levels, penicillamine therapy, benign or glandular fibroadenomas, mirror syndrome, and rarely malignancies, such as non-Hodgkin lymphoma (NHL), have been reported (1).

NHL is an extremely rare condition during pregnancy, especially considering that the primary breast lymphoma (PBL) accounts for $0.04 \%-$ $0.5 \%$ of primary breast tumors (2). The most frequent histopathologic types are diffuse large B-cell lymphoma, which accounts for up to $50 \%$ of all PBLs, $15 \%$ of follicular lymphoma, $12.2 \%$ of mucosa-associated lymphoid tissue lymphoma, and $10.3 \%$ of B-cell lymphoma (BL) and Burkitt-like lymphoma (3).

Normally, breast lymphoma presents as a unilateral painless breast masses in older women (average age at diagnosis, 55-60 years old) and is usually a B-cell NHL (4). A less common but distinctive presentation occurs in young women during or immediately after pregnancy affected by Burkitt lymphoma (5). Only 14 cases of Burkitt lymphoma with breast involvement during pregnancy have been reported so far (6). Herein, we describe the case of a young woman presenting at 40 weeks of gestation with unilateral gigantomastia, which was finally diagnosed as a Burkitt lymphoma.

\section{Case Presentation}

A 30-year-old gravida 1, para 0, woman was admitted at 40 weeks of gestation with a history of rapidly enlarging right breast at 2 weeks prior to presentation. Medical and obstetric histories were normal. General physical examination was unremarkable. Her breasts were asymmetrical, with the right breast larger, more tense, and more edematous than the left breast, which appears normal. No axillary adenopathy was noted. At admission to our hospital, she was diagnosed with mastitis and prescribed antibiotics. Because of non-response to the therapy, considering that the pregnancy was at term, delivery was considered. Vaginal stimulation by dinoprostone $10 \mathrm{mg}$ was carried out. After $24 \mathrm{~h}$ from induction, the patient delivered vaginally an infant weighing 3,110 g with Apgar scores of 9 and 10 at 1 and 5 min, respectively. 
After delivery, cabergoline $2 \mathrm{~g}$ was administered to block lactation, and a dose of $0.5 \mathrm{mg}$ pro die was administered in the following days. Despite the therapy and delivery, after 4 days, the right breast continued to grow until it was more than double the size of the left breast. Mastitis diagnosis was abandoned, and various imaging tests were done. Breast ultrasonography showed a widespread hyperplastic aspect of the mammary gland with evidence of bulky deep pseudonodular areas without clear signs of inflammation or fluid collections and absence of significant lymphadenopathy to the right axillary cavity. A dual-energy non-contrast computed tomography was performed, and the diagnostic hypothesis was unilateral gigantomastia due to a possible abnormal response to hormonal stimuli during pregnancy. To complete the imaging evaluation, the patient underwent bilateral contrast-enhanced magnetic resonance imaging (MRI) that revealed considerable increase in the size of the right breast $(170 \times 132 \times 180$ $\mathrm{mm}$ ) with a hyperplastic mainly fibroglandular mammary structure, interstitial stroma hypertrophy, and accentuated breast background parenchymal enhancement pattern that concluded with benign findings of right gestational macromastia. Considering the range of differential diagnoses for a woman presenting with gigantomastia in pregnancy, a thorough workup was performed, including white blood cell count, hematocrit, platelet count, electrolyte panel, hormone profile (estrogen, progesterone, and prolactin), liver function tests, serum calcium and albumin, and autoimmune tests (anti-dsDNA, antinuclear antibodies, rheumatoid factor, anti-Smith, cyclic citrullinated peptide, antithyroglobulin, and anti-thyroperoxidase). Results of these examinations were negative (7).

Objective measurement of the breast size and chest circumference was always performed by the same operator daily. As there was no clinical response, supported by the evaluation of the benignity in the instrumental investigations, medical therapy was continued. One week after the delivery, from the comparison with literature data, it was decided to replace cabergoline $0.5 \mathrm{mg} /$ die therapy with bromocriptine $2.5 \mathrm{mg}$ twice a day.

After 1 week of bromocriptine therapy, there was an increase in breast size (approximately $2 \mathrm{~cm}$ for each size), a variation of the chromatic characteristics of the skin, and progressing pain (Figure 1).

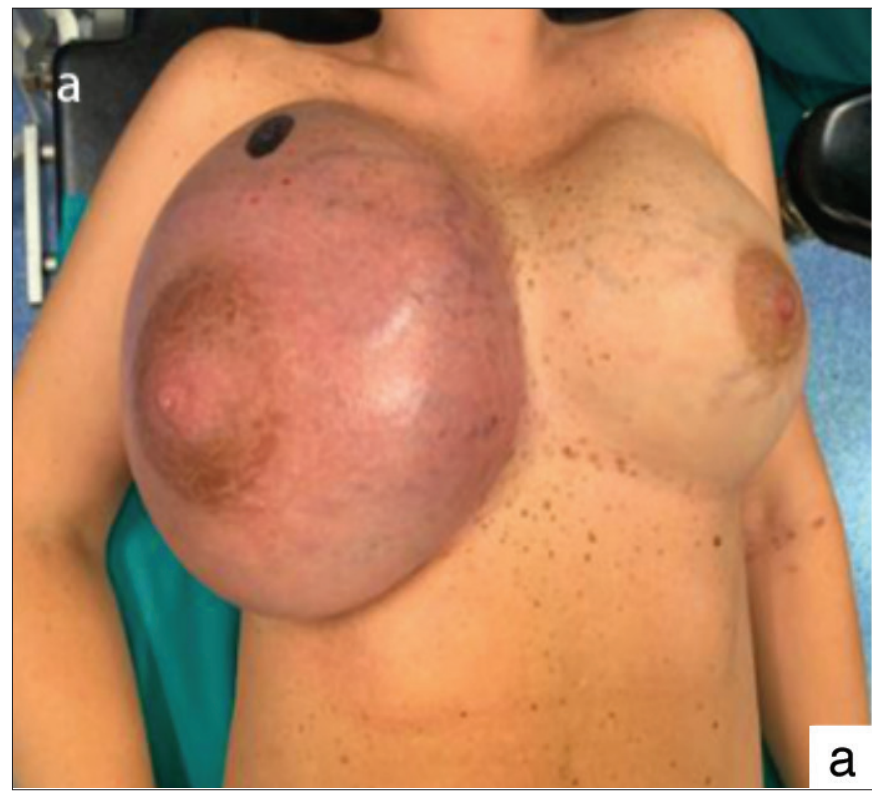

Breast MRI was repeated and demonstrated structural subversion of the mammary gland on both sides with a significant increase in the size on the right breast. In the right breast, an increase in glandular vasculature with ectasia of surface vessels was noted. Pathological lymph nodes in the axilla were also found (Figure 2).

\section{Key Points}

- Systemic malignancies should be considered in the differential diagnosis of gigantomastia during pregnancy.

- Burkitt lymphoma affecting the breasts during pregnancy or lactation is a rare entity that requires prompt diagnosis and an aggressive therapeutic approach.

- The best treatment approach for diffuse large BL is a combination of radiation, intensive chemotherapy, and limited surgical resection.

Since a tumor diagnosis could not be excluded, a preoperative biopsy of the right breast was performed. The main objective of this procedure was to have a histological confirmation to determine the correct therapeutic approach. The frozen section procedure revealed an NHL, so the surgery was stopped, and chemotherapy was initiated for patient management.

The final histological diagnosis revealed a sporadic Burkitt lymphoma with a characteristic "starry sky" pattern (Figure 3a, b).

Positron emission tomography/computed tomography showed intense fluorodeoxyglucose metabolism in both breasts, greater impairment of the right breast, involvement of some axillary lymph nodes, bilaterally retropectoral involvement, and right internal mammary chain involvement, as well as a widespread osteomedullary metabolic activation. Bone marrow aspiration and biopsy confirmed the presence of malignant cells. Thus, considering the evidence of disseminated lymphoma at the time of diagnosis, a systemic Burkitt lymphoma was defined. Wiseman and Liao's criteria for the diagnosis of primary NHL of the breast were not applicable (8). The patient was found to be suffering from stage IV NHL. A therapy with deltacortene $25 \mathrm{mg}$ twice a day and allopurinol $300 \mathrm{mg} /$ day was initiated, and in the meantime, the patient was transferred to a hematological referral center where

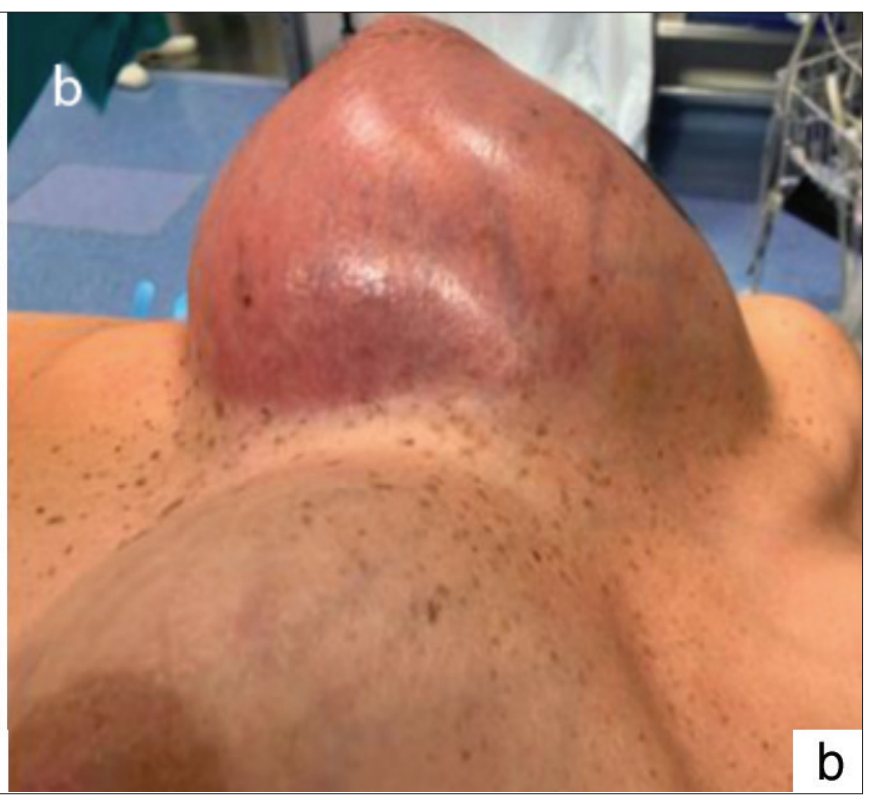

Figure 1. a, b. Right breast of the patient before the biopsy 


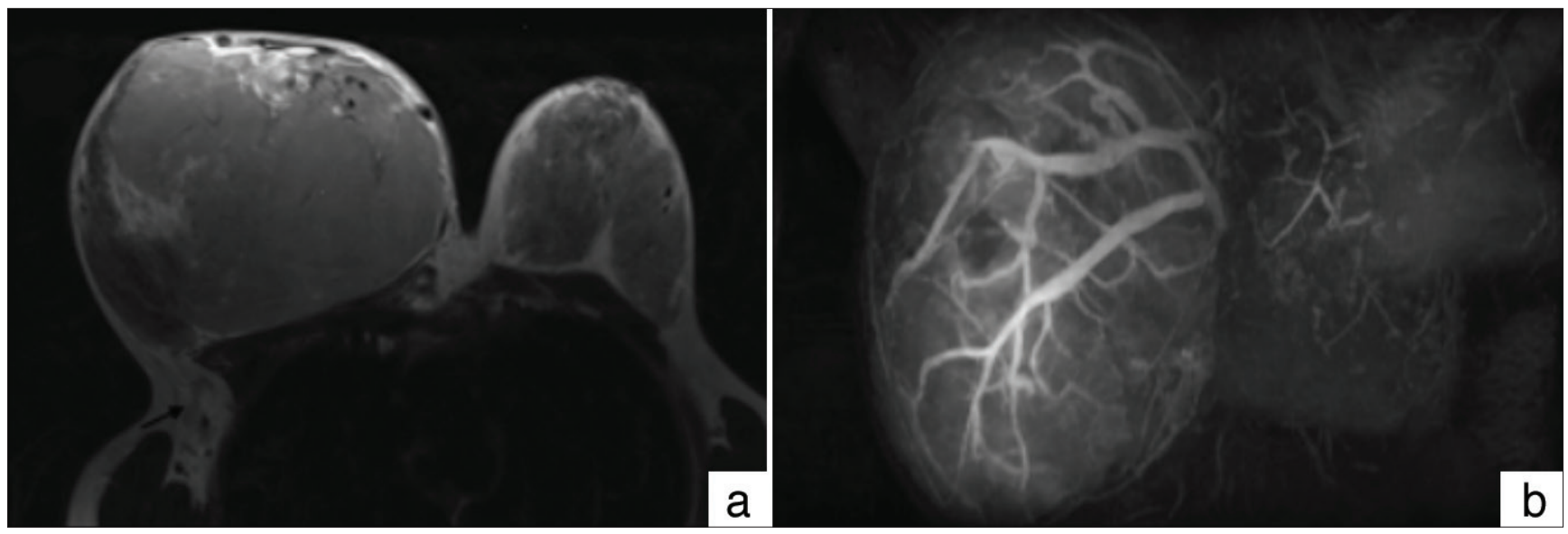

Figure 2. a, b. Mammary magnetic resonance (MMR) imaging with surface phase-array coil, turbo spin-echo axial T2 sequence. Structural subversion of the mammary gland on both sides, with a significant increase in the size of the right breast. Pathological lymph nodes (arrow) are found in the axilla (a). MMR with phase-array surface coil, T1 fl3d sequence after intravenous administration of contrast medium, MIP coronal reconstruction. Increase in glandular vasculature with ectasia of the surface vessels in the right breast (b)

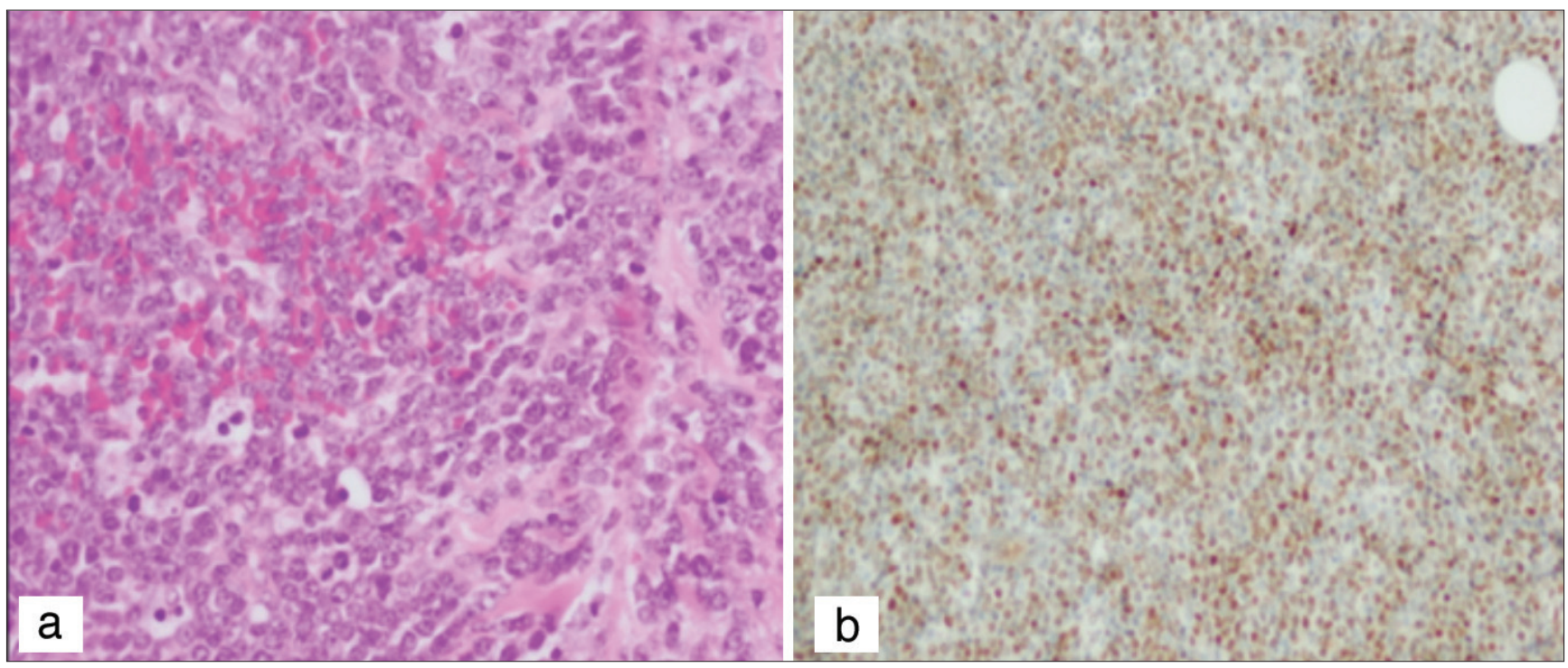

Figure 3. a, b. Microscopically, the morphological picture shows the absence of breast tissue replaced by monomorphic proliferation of medium-large polygonal cells, with an eccentric nucleus, dispersed chromatin, and 1-2 evident nucleoli with little cytoplasm and associated "starry sky" phenomenon (hematoxylin and eosin staining, $\times 40$ ) (a). On immunohistochemistry, the lymphoma cells are positive for c-MYC (×40) (b)

she has begun chemotherapy with a regimen of etoposide, prednisone, vincristine, cyclophosphamide, and doxorubicin plus the monoclonal antibody rituximab (9).

The patient gave her written informed consent before the procedure was performed.

\section{Discussion and Conclusion}

This case demonstrates that systemic malignancies such as diffuse large BL should be considered in the differential diagnosis of gigantomastia during pregnancy. Burkitt lymphoma affecting the breasts during pregnancy or lactation is a rare entity that requires prompt diagnosis and an aggressive therapeutic approach. Although there is no general agreement regarding the treatment of $\mathrm{BL}$ that involves the breast(s), avoided. The best treatment approach is a combination of radiation therapy, intensive chemotherapy, and limited surgical resection (10).

Recent literature reveals that the outcome of women with $\mathrm{BL}$ (considering all sites of presentation, including the liver, head, neck, abdomen, nodes, and breast) has improved since 1998, when the trend has been to treat $\mathrm{BL}$ in pregnant women aggressively with multiagent chemotherapy (10).

The implementation of more aggressive chemotherapy regimens in these patients could be a good way to handle this malignancy. Malignant causes of unilateral gigantomastia in pregnancy, such as NHL, should be considered in the differential diagnosis of this condition.

Informed Consent: Written informed consent was obtained from patient who participated in this case. 
Peer-review: Externally peer-reviewed.

\section{Author Contributions}

Concept: V.F.; Design: L.D.C.; Supervision: L.S.; Resources: C.S.; Materials: B.C.; Data Collection and/or Processing: G.C.; Analysis and/or Interpretation: L.D.C.; Literature Search: C.S.; Writing Manuscript: V.F.; Critical Review: L.S.; Other: B.C., G.C.

Conflict of Interest: The authors have no conflicts of interest to declare.

Financial Disclosure: The authors declared that this study has received no financial support.

\section{References}

1. Mangla M, Singla D. Gestational gigantomastia: A systematic review of case reports. J Midlife Health 2017; 8: 40-44. (PMID: 28458479) [Crossref]

2. Mattia AR, Ferry JA, Harris NL. Breast lymphoma. A B-cell spectrum including the low-grade B-cell lymphoma of mucosa associated lymphoid tissue. Am J Surg Pathol 1993; 17: 574-587. (PMID: 8333556) [Crossref]

3. Joks M, Myşliwiec K, Lewandowski K. Primary breast lymphoma: A review of the literature and report of three cases. Arch Med Sci 2011; 7: 27-33. (PMID: 22291729) [Crossref]
4. Janbabai G, Kayedimajd S, Alian S, Naghshvar F, Rashidi M, Farazmandfar T. Bilateral breast swelling in a 23-year-old woman with Burkitt lymphoma. J Res Med Sci 2012; 17: 1188-1191. (PMID: 23853639) [Crossref]

5. Giardini R, Piccolo C, Rilke F. Primary non-Hodgkin's lymphomas of the female breast. Cancer 1992; 69: 725-735. (PMID: 1730123) [Crossref]

6. Savvari P, Matsouka C, Barbaroussi D, Christoulas D, Nikitas N, Dimopoulos MA, et al. Burkitt's lymphoma in pregnancy with bilateral breastinvolvement: case report with review of the literature. Onkologie 2010; 33: 461-464. [Crossref]

7. Rezai S, Nakagawa JT, Tedesco J, Chadee A, Gottimukkala S, Mercado R, et al. Gestational gigantomastia complicating pregnancy: a case report and review of the literature. Case Rep Obstet Gynecol 2015; 2015: 892369. (PMID: 26713166) [Crossref]

8. Wiseman C, Liao KT. Primary lymphoma of the breast. Cancer 1972; 29: 1705-1712. (PMID: 4555557) [Crossref]

9. Gastwirt JP, Roschewszvki M. Management of adults with Burkitt lymphoma. Clin Adv Hematol Oncol 2018; 16: 812-822. (PMID: 30843890) [Crossref]

10. Hurley P, Linden MA, Peterson B, Blaes A. Burkitt lymphoma in pregnancy: two cases of successful Hematol treatment and continued fertility; with a review of the literature. Clin Lymphoma Myeloma Leuk 2013; 13: e10-e14. (PMID: 24021676) [Crossref] 